\section{Psychological Medicine}

cambridge.org/psm

\section{Original Article}

Cite this article: Hansson Bittár N, Falkstedt D, Sörberg Wallin A (2020). How intelligence and emotional control are related to suicidal behavior across the life course - $A$ registerbased study with 38-year follow-up.

Psychological Medicine 50, 2265-2271. https:// doi.org/10.1017/S0033291719002423

Received: 5 December 2018

Revised: 25 July 2019

Accepted: 20 August 2019

First published online: 9 October 2019

Key words:

Emotional control; intelligence; life course; neuroticism; suicide

Author for correspondence:

Nora Hansson Bittár

E-mail: nora.hansson@gmail.com

\title{
How intelligence and emotional control are related to suicidal behavior across the life course - $A$ register-based study with 38-year follow-up
}

\section{Nora Hansson Bittár ${ }^{1}$, Daniel Falkstedt ${ }^{2}$ and Alma Sörberg Wallin² ${ }^{2}$} ${ }^{1}$ Department of Clinical Neuroscience, Karolinska Institutet, Stockholm, Sweden and ${ }^{2}$ Department of Public
Health Sciences, Karolinska Institutet, Stockholm, Sweden (c) The Author(s) 2019. This is an Open Access article, distributed under the terms of the Creative Commons Attribution licence (http:// creativecommons.org/licenses/by/4.0/), which permits unrestricted re-use, distribution, and reproduction in any medium, provided the original work is properly cited.

\section{CAMBRIDGE} UNIVERSITY PRESS

\section{Abstract}

Background. Both low intelligence and low emotional control have previously been linked to a higher risk of suicide, but it is unknown whether the associations apply consistently over the life course.

Methods. The study was based on data on intelligence and emotional control, collected from 48738 Swedish men conscripted in 1969-1970, at ages 18-20 years. The data were linked to national registers giving information on subsequent suicidal behavior (completed and attempted suicide) up to the age of 59 years. The associations were investigated using logistic regression and Cox proportional hazards regression models, with adjustment for childhood socioeconomic status.

Results. Intelligence and emotional control assessed in late adolescence both showed robust inverse associations with suicidal behavior over the 38-year follow-up. However, while the association between lower intelligence and higher rate of suicidal behavior remained the same throughout ( $\sim 40 \%$ increased hazard per unit on a five-level scale), the association between lower emotional control and suicidal behavior was substantially stronger in early adulthood ( $\sim 100 \%$ increased hazard per unit) than in late middle age ( $\sim 30 \%$ increased hazard per unit).

Conclusions. The study adds to previous research by showing that the association between poor emotional control and subsequent suicide risk in men becomes weaker over the life course, while the association between low intelligence and suicide risk seems to be constant. The particularly high suicide risk of young men with poor emotional control may motivate targeted prevention efforts.

\section{Introduction}

Suicide is one of the leading causes of death worldwide, and approximately one million people die each year from suicide (World Health Organization, 2012). Like many other health-related outcomes, suicide is not the consequence of a single disease factor. Rather, there are multiple factors that contribute to completed and attempted suicide (here denoted as suicidal behavior), such as the presence of psychiatric disorders, physical illnesses, impulsive behaviors, and life stressors (Gunnell and Lewis, 2005). Previously, it has been shown that both lower level of intelligence and higher level of neuroticism, or low emotional control, are related to an increased risk of suicidal behavior (Allebeck et al., 1988; Brezo et al., 2006; Batty et al., 2010; Gravseth et al., 2010; Sörberg et al., 2013). However, little is yet known about these associations from a lifecourse perspective, with regards to whether the associations change or remain stable over time.

Intelligence is a general ability that reflects a broad and deep capability of understanding our surroundings, which involves the ability to reason, plan, solve problems, think abstractly, comprehend complex ideas, learn quickly, and learn from experience (Gottfredson, 1997). Research in recent years has provided extensive evidence that intelligence is an important predictor of major life outcomes, such as educational attainment, occupation, and health, and also suicidal behavior (Batty et al., 2010; Sörberg et al., 2013; Plomin and Deary, 2015). Moreover, intelligence is fairly stable over the life course. For example, in a Scottish cohort of about 1000 women and men, the raw stability coefficient for people who completed the same intelligence test at age 11 and age 70 was on average 0.67 (Gow et al., 2011).

Emotional control is a construct aimed to capture factors such as susceptibility to stress, anxiety, psychosomatic symptoms, and poor control over nervousness and aggression (Potijk et al., 2016). This construct has not been widely used in research on suicidal behavior but shows great similarities with one of the big five personality dimensions, namely neuroticism, which is associated with anxiety, anger, depression, impulsiveness and vulnerability (Chamorro-Premuzic, 2016). Although emotional control and neuroticism are not identical 
constructs, they are highly overlapping. Both concern stable personality traits related to emotional stability, and both have been shown to be associated with an increased risk of suicidal behavior (Allebeck et al., 1988; Brezo et al., 2006; Wiktorsson et al., 2013). The societal economic burden attributed to neuroticism exceeds that of common mental disorders (Cuijpers et al., 2010).

Neuroticism has been found to be mostly stable over the life course, but, according to a meta-analysis, a decline in the population mean of neuroticism takes place during late adolescence and early adulthood (Roberts et al., 2006). After the age of 30, however, the level of neuroticism tends to regain stability at the group level (Roberts et al., 2006). Specifically, test-retest consistency increases with age from 0.45 during adolescence to 0.60 during young and middle adulthood (Roberts and DelVecchio, 2000).

Even though both lower intelligence and higher neuroticism have previously been linked to an increased risk of subsequent suicidal behavior (Allebeck et al., 1988; Brezo et al., 2006; Batty et al., 2010; Gravseth et al., 2010; Sörberg et al., 2013), little is yet known about those associations from a life-course perspective. Risk factors for suicide might act differently over the life course (Gunnell and Lewis, 2005) and, likewise, the roles of intelligence and emotional control might change over time. Indeed, we have found previously that the association between intelligence measured at age 18-20 and subsequent depression was particularly strong in young adulthood, but then attenuated considerably towards late middle age (Lager et al., 2017). Previous researchers have stressed that knowledge of risk factors is essential for the effective prevention of suicidal behavior (Gunnell and Lewis, 2005; Nock et al., 2008). From that perspective, understanding of the long-term associations between individual differences and suicidal behavior should be of greatest interest.

\section{Aim}

The aim of this study was to investigate how the associations between intelligence and emotional control in early adulthood and subsequent suicidal behavior develop over time. We examined those associations in a Swedish male cohort with measurements from military conscription in 1969-1970. The cohort information was linked to national register data on suicidal behavior, with childhood socioeconomic status (SES) treated as a potential confounder, and followed over 38 years, up to the age of 59 .

\section{Method}

\section{Study population}

The study was based on data collected at compulsory conscription for Swedish military service in 1969 and 1970. About 97-98\% of all Swedish men were conscripted at that time; the remaining 2-3\% were in most cases exempted due to severe disability or congenital disorders. The cohort consisted of 49321 men born 1949-1951 (aged 18-20). Approximately 2\% of all the men who were conscripted during those years were born prior to 1949 , and were excluded from the data set in order to obtain as homogeneous a cohort as possible. All information was anonymized before it was made available for research to protect the integrity of the individuals. The research has been approved by the ethical committee at Karolinska Institutet (numbers 2004/5:9-639/5 and 2010/604:32).

From the original cohort, 583 men were excluded due to missing data on intelligence $(n=75)$ and/or emotional control
( $n=396)$, and/or death or emigration prior to the start of follow-up ( $n=175)$, which left 48738 men (98.8\%) for analysis.

\section{Material and procedure}

\section{Conscription in Sweden}

The Swedish conscription process included physical and medical assessments, a test of intelligence, an interview with a psychologist, and questionnaires that covered social background, psychological and psychosomatic problems, and adjustment and substance use (Allebeck et al., 1988).

\section{Intelligence}

Intelligence was measured at conscription through a multidimensional test, which included four subtests examining verbal, logic-inductive and visuospatial ability, and technical comprehension. The outcomes were converted into a global score with a normal (Gaussian) distribution on a nine-level scale. The test battery was designed to measure general intelligence, and used primarily to find a position with appropriate learning demands and predict the conscript's ability to profit from education within the military (Ross, 1988). A detailed description of the test is presented elsewhere (Karlsson et al., 2010; Sörberg Wallin, 2015). Test-retest reliability was 0.81 for the global score of a group of 107 men retested after one to three years (Ross, 1988). In the current study, the nine-level scale was converted into a five-level scale in order to match the scale of emotional control. Following conversion, the scale approximately corresponded to IQ bands of $<82,82-95,96-110,111-126$, and $>126$.

\section{Emotional control}

Assessment of emotional control was made through a semistructured interview administered by a trained psychologist. The interview took about 20-30 min, and a summary of the interview process is provided elsewhere (Potijk et al., 2016; Ross, 1988). The overall aim of the interview was to measure the conscript's suitability for military service, including tolerance of mental stress. Emotional control was measured as situation-specific regulation of emotions. The psychologist asked the conscripts how they emotionally responded to important events and situations experienced in childhood and adolescence, for example personal conflicts or stress at school or work. Based on the responses, the psychologists rated emotional control on a scale ranging from one (very low) to five (very high). Higher scores on emotional control were allocated to conscripts with high stress tolerance, low anxiety and control over nervousness and aggression, whereas lower scores were allocated to subjects with psychosomatic symptoms, low levels of stress tolerance, anxiety and problems in controlling nervousness and aggression (Stenbacka and Jokinen, 2015). The inter-rater reliability of the psychologists' ratings has been found to be as high as 0.85 (Lilieblad and Ståhlberg, 1977).

\section{Suicidal behavior}

The conscription data were linked to register data on suicidal behavior, which encompasses both completed and attempted suicide. Information about completed suicides during the period 1973-2008 was collected from the National Cause of Death Register, and information about suicide attempts for the same time period was obtained from the National Hospital Discharge Register. The variables were classified following the International Classification of Disease (ICD) codes for suicide and suicide attempt (ICD-8/9: E950-9; ICD-10: X60-X84), and for events of 
undetermined intent (ICD-8/9: E980-9; ICD-10: Y10-Y34). The latter was included since previous research has shown that most deaths from events of undetermined intent are likely to be suicides (Jiang et al., 2014).

\section{Socioeconomic status}

Information on childhood SES was collected from the national census in 1960. The SES categories were based on the occupation of the father, if available, but otherwise the mother. The six categories were: (1) unskilled workers, (2) skilled workers, (3) assistant non-manual employees, (4) non-manual employees at intermediate or higher level, (5) farmers, and (6) others or those with no occupation reported.

\section{Statistical analysis}

The associations between intelligence and emotional control at conscription and subsequent suicidal behavior were first estimated for the full follow-up period using Cox proportional hazard models, yielding hazard ratios (HRs) with 95\% confidence intervals (CIs). The conscripts were followed until emigration, death, or the first event of suicidal behavior, or until the end of 2008 , whichever came first.

The follow-up period was then divided into four approximate decades (1973-1979, 1980-1989, 1990-1999, and 2000-2008). Thereafter, logistic regressions yielding odds ratios (ORs) were used to investigate the associations of intelligence and emotional control with suicidal behavior for each decade separately in the men who were alive and had not emigrated at the start of each period. Tests of interaction with time were performed to test formally whether the associations remained stable over time. The independent variables, intelligence and emotional control, were modeled as continuous variables, with HRs and ORs given for each step decrease on the scales of intelligence and emotional control, and also as categorical variables to obtain HRs and ORs for each level, with the highest levels as the reference categories. All analyses were adjusted for childhood SES. We then repeated the analyses with mutual adjustment for intelligence and emotional control to separate their associations with suicidal behavior from each other.

Additionally, we performed sensitivity analyses by excluding completed suicide from the dependent variable, and also by excluding events with undetermined intent. These analyses were performed to check whether the results were robust to any differences between attempted and completed suicide and between undetermined and certain suicidal intent. All analyses were performed using SAS 9.4 (SAS Institute, Inc., Cary, NC, USA 2012).

\section{Results}

During the total follow-up period (1973-2008), 1725 of the men (3.5\%) had a suicidal-behavior event (493 completed and 1232 attempted suicides as their first event). The numbers of men with a suicidal-behavior event across the levels of intelligence and emotional control are shown in Table 1.

The associations of intelligence and emotional control with suicidal behavior followed dose-response patterns, as shown in Table 1 (parts a and c, IQ and EC in units, modelled as categorical variables). For example, men in the lowest intelligence category (1) had a six-fold higher risk of suicidal behavior (HR and 95\% CI 6.29, 4.48-8.83), and men in the second lowest category (2) had a four-fold higher risk of suicidal behavior (4.02,
2.89-5.60), compared with men in the highest intelligence category (5). In a similar manner, men in the lowest category of emotional control (1) had an almost seven-fold higher risk of suicidal behavior $(6.64,4.75-9.28)$, and men in the second lowest category (2) had a three-fold higher risk of suicidal behavior (3.46, 2.50-4.78), compared with men in the highest category (5).

When modeling intelligence and emotional control as continuous variables (parts b and d in Table 1, IQ and EC per unit decrease), suicidal behavior between 1973 and 2008 was associated with both lower intelligence (HR and 95\% CI 1.54, 1.461.61 per unit decrease on the five-level scale) and lower emotional control $(1.68,1.60-1.76)$, after adjustment for childhood SES. This implies that one unit lower intelligence on the five-level scale was associated with a $54 \%$ higher risk of suicidal behavior and one unit lower emotional control was associated with a $68 \%$ higher suicidal risk. In the sensitivity analyses (both modeling intelligence and emotional control as continuous variables, per unit decrease; not shown in the table), when completed suicide was excluded from the dependent variable, the HR for intelligence (1.61, 1.52-1.70 per unit decrease) and the HR for emotional control (1.77, $1.67-1.88$ per unit decrease) were only slightly higher; and excluding events of undetermined intent gave quite similar results to those of the main analysis $(1.57,1.47-1.67$ for intelligence, and 1.73, 1.62-1.86 for emotional control, per unit decrease). Further, a crude analysis without controlling for childhood SES yielded very similar results $(1.53,1.46-1.60$ for intelligence and 1.70, 1.61-1.78 for emotional control, per unit decrease), implying that childhood SES was not a confounder that would explain the associations.

When analyzed over the separate follow-up periods, the association between intelligence and suicidal behavior remained fairly stable over the life course, whereas the association between emotional control and suicidal behavior gradually weakened, see Table 1 (parts $\mathrm{b}$ and $\mathrm{d}$; the continuous variables were used in these models). For example, one lower unit of intelligence was associated with a $65 \%$ higher risk of suicidal behavior during the first follow-up period (OR and 95\% CI 1.65, 1.49-1.82) and a 53\% higher risk of suicidal behavior during the last follow-up period $(1.53,1.39-1.69)$. On the other hand, one lower unit of emotional control was associated with a $117 \%$ higher risk of suicidal behavior during the first follow-up period $(2.17,1.95-2.40)$ but with only a $44 \%$ higher suicidal risk during the fourth and last follow-up period (1.44, 1.30-1.59). A significance test confirmed that the association with emotional control weakened over the follow-up period ( $p<0.0001$ for interaction with time). In the models with mutual adjustments for intelligence and emotional control, the associations were weakly to moderately attenuated but the difference between them over the total follow-up period remained, see Fig. 1. The exact estimates and 95\% CIs are shown in online Supplementary Table S1.

\section{Discussion}

In sum, our analyses showed that the association between low intelligence and suicidal behavior was fairly constant over the follow-up period to age 59 in this cohort of Swedish men, whereas the association between low emotional control and suicidal behavior weakened with increasing age. Mutual adjustment for intelligence and emotional control attenuated the associations somewhat but did not attenuate the difference between them. Adjustment for socioeconomic status during childhood had only marginal effects on the results. 
Table 1. Associations of intelligence (IQ) and emotional control (EC) in 1969/1970 with suicidal behavior 1973-2008

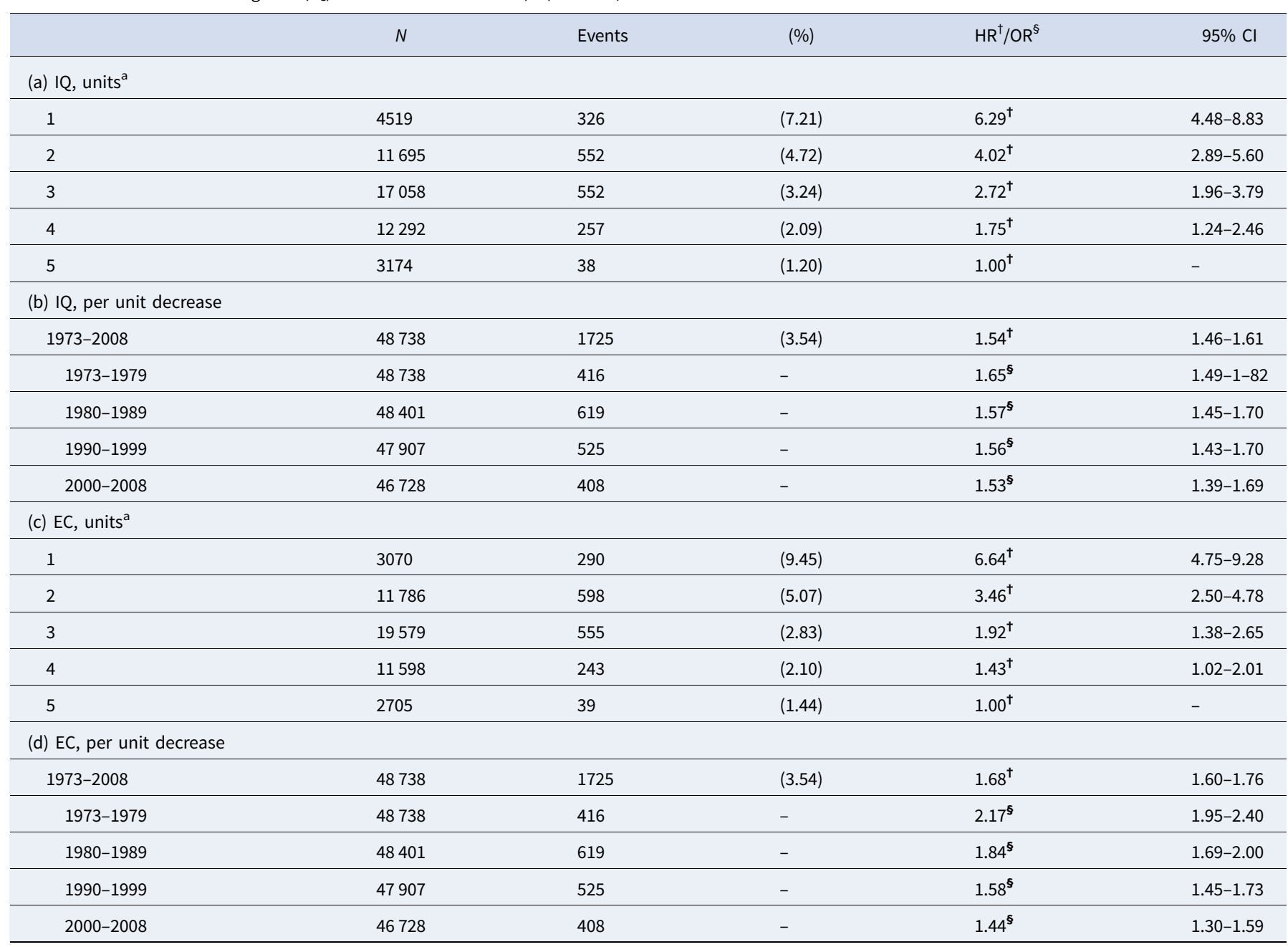

Parts a and c: Hazard ratios (HRs; marked with “'’) with 95\% confidence intervals ( $\mathrm{Cls}$ ) for the entire follow-up period, modelling IQ and EC as categorical variables. Parts b and d: HRs for the entire follow-up and odds ratios (ORs; marked with ' ${ }^{\text {(5) }}$ ) for the separate time periods, with 95\% Cls, modelling IQ and EC as continuous variables. All models are adjusted for childhood SES ${ }^{a}$ One (1) represents the lowest level and five (5) the highest level of intelligence and emotional control. Level 5 is the reference category in these analyses (HR=1)

Fig. 1. Associations between suicidal behavior and intelligence and emotional control, respectively, with adjustment for each other and for childhood SES. ORs with $95 \% \mathrm{Cls}$ for suicidal behavior are given for a one step decrease on the five-level scales of intelligence and emotional control for the separate follow-up periods. The time periods end at about 10, 20, 30 and 40 years after conscription, respectively, and correspond with approximate age intervals of 22-28 years, $29-38$ years, $39-48$ years and $49-58$ years.

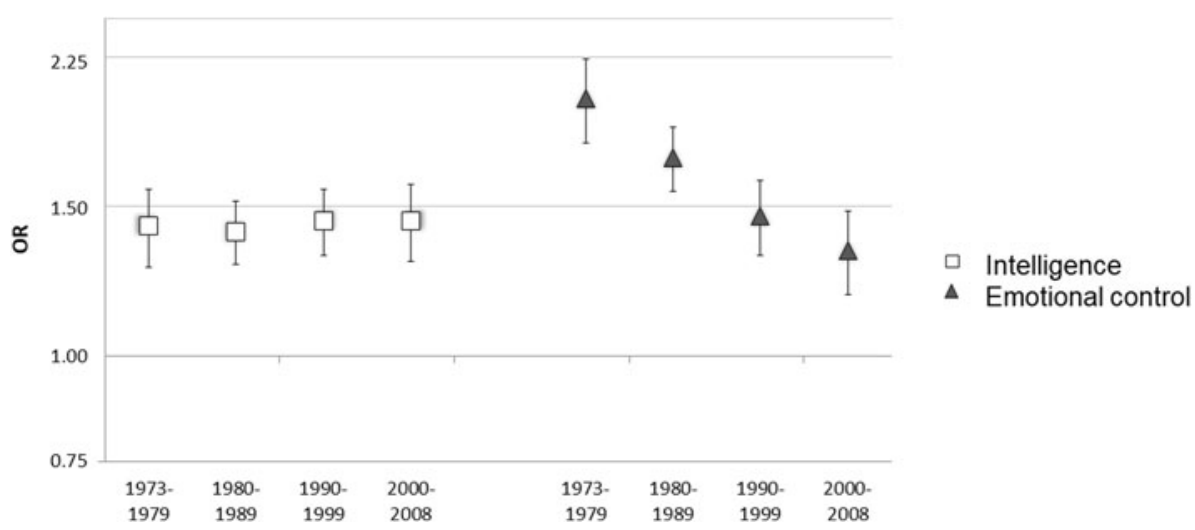

\section{Findings in relation to previous research}

Suicidal behavior has been linked to lower intelligence and higher neuroticism (which is similar to low emotional control) in some previous studies (Brezo et al., 2006; Osler et al., 2008; Gravseth et al., 2010; Wiktorsson et al., 2013), including a few separate studies of the same cohort of men as in the current study (Allebeck et al., 1988; Batty et al., 2010; Sörberg et al., 2013).
We are not aware of any previous studies focusing on changes in these associations over the life course, but a previous study using the same cohort showed that lower intelligence and a higher risk of hospitalization for major depression had a weakening association over the 38-year follow-up (Lager et al., 2017). Since depression is a well-known risk factor for suicide (Lesage et al., 1994), it is somewhat surprising that we found the association between higher intelligence and lower risk of suicidal behavior 
to remain stable over the life course. Nevertheless, risk factors for suicidal behavior are not identical to those for psychiatric disorders and their impact during the life span may differ (Gunnell and Lewis, 2005), which might explain the divergent findings.

\section{Possible explanations and implications}

Although the associations of both intelligence and emotional control with suicidal behavior remained after adjustment, it is not necessarily the case that low intelligence and low emotional control are immediate causes of suicidal behavior. To some extent, these psychological differences between individuals may also predict suicidal behavior along indirect pathways. Several potential mechanisms underlying the associations of intelligence and neuroticism with suicidal behavior have been presented in previous research. For example, Gottfredson and Deary (2004) stress that intelligence may enhance individuals' care for their own health, since it represents learning, reasoning and problem-solving skills. Further, lower neuroticism (Gunthert et al., 1999), and presumably also higher intelligence, are associated with more adaptive coping strategies, which might increase resilience in the face of stressful life events and thereby prevent suicidal behavior (Andersson et al., 2008; Wiktorsson et al., 2013). Also, it has been suggested that individuals with high neuroticism are more likely than others to experience stressful life events, and also gain less social support (Kendler et al., 2003; Deary et al., 2010), which may help to explain their increased risk of suicidal behavior.

One of the main findings of this study was that the association between intelligence and suicidal behavior remained constant over the entire follow-up period, while the association between emotional control and suicidal behavior was particularly strong in young adulthood and then weakened across the four periods. Largely, the two characteristics were associated with suicidal behavior independently of each other, as shown in the mutually adjusted models. Each, however, accounted for some of the other characteristic's association with suicidal behavior: for emotional control to a greater extent in the first follow-up periods, and for intelligence to an increasing extent in the later periods. Thus, mutual adjustment did not reduce the difference in time trends between intelligence and emotional control.

The gradual weakening of the association between emotional control and suicidal behavior may have some different potential explanations. One is that emotional control is more variable than intelligence from a life-course perspective. This idea is supported by previous research showing that there is a decrease in the population mean of neuroticism during late adolescence while intelligence seems to be more stable (Roberts et al., 2006). Similarly, previous research has shown that the presence of negative emotions, such as stress and anger, steeply decline from the early 20s, and that older adults are less emotionally reactive to stressors and less engaged in destructive conflict strategies than younger adults (Stone et al., 2010; Mather, 2012). Another potential explanation for why the association weakens is that stressful circumstances or life events during the years leading up to the conscription examination, e.g. in school (Lager et al., 2016), could both have had a negative impact on emotional control, as rated in the psychologists' interviews at conscription, and increased the incidence of suicidal behaviors for a time following the conscription examination. This would be consistent with findings showing that some of the fluctuations in neuroticism over time seems to be associated with life events (Ormel et al.,
2012). In principle, however, the conscription interview was designed to measure a personality trait, not an emotional state.

Our results highlight the possibility that low intelligence and less surprisingly - poor emotional control influence the risk of suicide. The importance of this for suicide-preventive work may be worth investigating further. Other studies have discussed how low intelligence and poor emotional control also increase the likelihood of ending up in difficult circumstances, e.g. with low education or problematic social relations (Roberts et al., 2007; Deary et al., 2010). In accordance with this, studies in which we ourselves were involved have suggested that low socioeconomic status, and also risky behaviors, in young adulthood help to explain the relationships between intelligence, emotional control and health outcomes later in life (Sörberg et al., 2013; Potijk et al., 2016). In particular, our results may justify paying increased attention to the evidence that neuroticism and poor emotional control may contribute to high but eventually diminishing rates of harmful behaviors, including suicide, in young adult men. Access to treatment for the young may have to be given priority, since there is some evidence that neurotic problems can be reduced, e.g. with the help of cognitive behavioral therapy (CBT) and similar forms of psychotherapy (Jorm, 1989; Tang et al., 2009).

On the other hand, one possible reason for the long-term association between intelligence and suicidal behavior is that intelligence is robustly associated with attained education and socioeconomic position in adulthood (Plomin and Deary, 2015). These factors have been found to explain substantial proportions of the association between intelligence in youth and suicidal behavior in adulthood (Sörberg et al., 2013; Sörberg Wallin et al., 2018a). They may be relevant to prevention at the societal level, not least because socioeconomic factors contribute considerably to suicide risk at the population level (Li et al., 2011). Of note is that this pathway might be more important for suicide prevention in men than in women; in women, low intelligence seems to be associated with suicide attempt (Sörberg Wallin et al., 2018b) but not death from suicide (Andersson et al., 2008; Calvin et al., 2017; Sörberg Wallin et al., 2018a). The fact that gender differences are observed in these relationships further supports the hypothesis that social and socioeconomic factors are involved as mediators or moderators in the associations.

\section{Strengths and limitations}

There are several strengths to this study. The study population is strongly representative of the Swedish population of men who were born in 1950 and 1951, since nearly all men participated in compulsory conscription at that time. The exposure variables were measured using standardized test batteries and semistructured interviews following manuals, and the outcome variables are based on national records with high reliability (Lilieblad and Ståhlberg, 1977; Ross, 1988). Further, the 38-year follow-up period enabled us to follow the men in the cohort over a substantial part of their lives.

There are, however, two aspects of this study that limits its generalizability. First, we cannot generalize the results to women since only men were obliged to participate in conscription. Second, the results cannot be directly extrapolated to other settings partly because suicidal behavior differs between cultures (Nock et al., 2008). Therefore, generalizations to other populations should be made with these limitations in mind. Further, our outcome variable, suicidal behavior, includes both completed 
and attempted suicide. This merged variable has been used in previous studies of the same cohort (Åberg et al., 2014), but since suicide attempt is far more frequent than completed suicide (Leon et al., 1990), and behavior associated with completed suicide may differ from behavior associated with suicide attempt, the merger might seem debatable. However, because our data include only severe suicide attempts that have led to hospitalization, it can be assumed that attempted and completed suicides are quite similar in the present study. In our analyses, the estimates were only slightly higher when completed suicide was excluded from the dependent variable. Lastly, intelligence and emotional control were measured at one point in time only. While intelligence is quite stable over the life course (Gow et al., 2011), personality aspects such as emotional control are more variable. Data with repeated measures of personality aspects are needed to directly investigate how changes in these measures might explain changes in their relationship with suicidal behavior.

\section{Conclusions}

In line with previous research, this study confirms that low intelligence and low emotional control are both associated with a higher risk of suicidal behavior among men. Further, this study adds to previous research by showing that the association between intelligence and suicidal behavior seems constant over the life course, whereas the association between emotional control and suicidal behavior may become considerably weaker over time. The particularly high suicide risk of young men with poor emotional control may motivate targeted prevention efforts.

Supplementary material. The supplementary material for this article can be found at https://doi.org/10.1017/S0033291719002423

Acknowledgements. We would like to thank Tomas Hemmingsson for providing data and for helpful advice.

Financial support. This research was funded by Forte, grant numbers 2015-00057 and 2017-00173.

\section{Conflict of interest. None.}

Ethical standards. The authors assert that all procedures contributing to this work comply with the ethical standards of the relevant national and institutional committees on human experimentation and with the Helsinki Declaration of 1975 , as revised in 2008. Because of the character of the database, the normal requirement for written consent was waived. Instead, all data were anonymized before they were made available for research in order to protect the integrity of the individuals, and the data were handled in a careful and sensitive way. Further, studies of individual differences in intelligence, personality and suicide may be controversial because they highlight inequalities between individuals. However, gaining knowledge about how individual differences in intelligence and emotional control are associated with suicidal behavior will hopefully help us to reduce inequalities and prevent suicides in the long run.

\section{References}

Åberg MAI, Nyberg J, Torén K, Sörberg A, Kuhn HG and Waern M (2014) Cardiovascular fitness in early adulthood and future suicidal behaviour in men followed for up to 42 years. Psychological Medicine 44, 779-788.

Allebeck P, Allgulander C and Fisher LD (1988) Predictors of completed suicide in a cohort of 50465 young men: role of personality and deviant behaviour. British Medical Journal 297, 176-178.

Andersson L, Allebeck P, Gustafsson JE and Gunnell D (2008) Association of IQ scores and school achievement with suicide in a 40-year follow-up of a Swedish cohort. Acta Psychiatrica Scandinavica 118, 99-105.
Batty GD, Whitley E, Deary IJ, Gale CR, Tynelius P and Rasmussen F (2010) Psychosis alters association between IQ and future risk of attempted suicide: cohort study of 1109475 Swedish men. British Medical Journal 340, c2506.

Brezo J, Paris J and Turecki G (2006) Personality traits as correlates of suicidal ideation, suicide attempts, and suicide completions: a systematic review. Acta Psychiatrica Scandinavica 113, 180-206.

Calvin CM, Batty GD, Der G, Brett CE, Taylor A, Pattie A, Čukić I and Deary IJ (2017) Childhood intelligence in relation to major causes of death in 68 year follow-up: prospective population study. British Medical Journal 357, j2708.

Chamorro-Premuzic T (2016) Personality and Individual Differences (3rd ed.), pp. 53-54. Glasgow: John Wiley \& Sons.

Cuijpers P, Smit F, Penninx BW, de Graaf R, ten Have M and Beekman AT (2010) Economic costs of neuroticism: a population-based study. Archives of General Psychiatry 67, 1086-1093.

Deary IJ, Weiss A and Batty GD (2010) Intelligence and personality as predictors of illness and death how researchers in differential psychology and chronic disease epidemiology are collaborating to understand and address health inequalities. Psychological Science in the Public Interest 11, 53-79.

Gottfredson LS (1997) Mainstream science on intelligence: an editorial with 52 signatories, history, and bibliography. Intelligence 24, 13-23.

Gottfredson LS and Deary IJ (2004) Intelligence predicts health and longevity, but why? Current Directions in Psychological Science 13, 1-4.

Gow AJ, Johnson W, Pattie A, Brett CE, Roberts B, Starr JM and Deary IJ (2011) Stability and change in intelligence from age 11 to ages 70, 79, and 87: the Lothian Birth Cohorts of 1921 and 1936. Psychology and Aging 26, 232.

Gravseth HM, Mehlum L, Bjerkedal T and Kristensen P (2010) Suicide in young Norwegians in a life course perspective: population-based cohort study. Journal of Epidemiology and Community Health 64, 407-412.

Gunnell D and Lewis G (2005) Studying suicide from the life course perspective: implications for prevention. The British Journal of Psychiatry 187, 206-208.

Gunthert KC, Cohen LH and Armeli S (1999) The role of neuroticism in daily stress and coping. Journal of Personality and Social Psychology 77, 1087-1100.

Jiang GX, Hadlaczky G and Wasserman D (2014) Självmord i Sverige-Data: 1980-2013. Nationellt centrum för suicidforskning och prevention av psykisk ohälsa (NASP) vid Karolinska Institutet, Stockholm.

Jorm AF (1989) Modifiability of trait anxiety and neuroticism: a meta-analysis of the literature. Australian \& New Zealand Journal of Psychiatry 23, 21-29.

Karlsson H, Ahlborg B, Dalman C and Hemmingsson T (2010) Association between erythrocyte sedimentation rate and IQ in Swedish males aged 18-20. Brain, Behavior, and Immunity 24, 868-873.

Kendler KS, Gardner CO and Prescott CA (2003) Personality and the experience of environmental adversity. Psychological Medicine 33, 1193-1202.

Lager A, Seblova D, Falkstedt D and Lövden M (2016) Cognitive and emotional outcomes after prolonged education: a quasi-experiment on 320182 Swedish boys. International Journal of Epidemiology 46, 303-311.

Lager E, Melin B, Hemmingsson T and Wallin AS (2017) The evolving relationship between premorbid intelligence and serious depression across the lifespan-A longitudinal study of 43,540 Swedish men. Journal of Affective Disorders 211, 37-43.

Leon AC, Friedman RA, Sweeney JA, Brown RP and Mann JJ (1990) Statistical issues in the identification of risk factors for suicidal behavior: the application of survival analysis. Psychiatry Research 31, 99-108.

Lesage AD, Boyer R, Grunberg F, Vanier C, Morissette R and Loyer M (1994) Suicide and mental disorders: a case-control study of young men. The American Journal of Psychiatry 151, 1063.

Li Z, Page A, Martin G and Taylor R (2011) Attributable risk of psychiatric and socio-economic factors for suicide from individual-level, population-based studies: a systematic review. Social Science \& Medicine 72, 608-616.

Lilieblad B and Ståhlberg A (1977) Reliabilitet hos psykologiska bedömningar vid inskrivningsprovning. FOA-rapport $C$, 55011-H7.

Mather M (2012) The emotion paradox in the aging brain. Annals of the New York Academy of Sciences 1251, 33-49. 
Nock MK, Borges G, Bromet EJ, Cha CB, Kessler RC and Lee S (2008) Suicide and suicidal behavior. Epidemiologic Reviews 30, 133-154.

Ormel J, Riese H and Rosmalen JG (2012) Interpreting neuroticism scores across the adult life course: immutable or experience-dependent set points of negative affect? Clinical Psychology Review 32, 71-79.

Osler M, Andersen AN and Nordentoft M (2008) Impaired childhood development and suicidal behaviour in a cohort of Danish men born in 1953. Journal of Epidemiology and Community Health 62, 23-28.

Plomin R and Deary IJ (2015) Genetics and intelligence differences: five special findings. Molecular Psychiatry 20, 98-108.

Potijk MR, Janszky I, Reijneveld SA and Falkstedt D (2016) Risk of coronary heart disease in men with poor emotional control: a prospective study. Psychosomatic Medicine 78, 60-67.

Roberts BW and DelVecchio WF (2000) The rank-order consistency of personality traits from childhood to old age: a quantitative review of longitudinal studies. Psychological Bulletin 126, 3.

Roberts BW, Walton KE and Viechtbauer W (2006) Patterns of mean-level change in personality traits across the life course: a meta-analysis of longitudinal studies. Psychological Bulletin 132, 1.

Roberts BW, Kuncel NR, Shiner R, Caspi A and Goldberg LR (2007) The power of personality: the comparative validity of personality traits, socioeconomic status, and cognitive ability for predicting important life outcomes. Perspectives on Psychological Science 2, 313-345.

Ross A (1988) De värnpliktigas prestationsförmåga vid inskrivningspövningar $i$ Sverige 1969-1979 [The conscripts' capacity at enlistment testing in Sweden 1969-1979]. Umeå: Umeå Universitet, Pedagogiska Institutionen.

Sörberg A, Allebeck P, Melin B, Gunnell D and Hemmingsson T (2013) Cognitive ability in early adulthood is associated with later suicide and suicide attempt: the role of risk factors over the life course. Psychological Medicine 43, 49-60.

Sörberg Wallin A (2015) The Association of Intelligence with Morbidity, Mortality and Disability Pension: Epidemiological Studies in a Cohort of Swedish men. Stockholm: Institute of Environmental Medicine.

Sörberg Wallin A, Allebeck P, Gustafsson J-E and Hemmingsson T (2018a) Childhood IQ and mortality during 53 years' follow-up of Swedish men and women. Journal of Epidemiology and Community Health 72, 926-932.

Sörberg Wallin A, Zeebari Z, Lager A, Gunnell D, Allebeck $P$ and Falkstedt D (2018b) Suicide attempt predicted by academic performance and childhood IQ: a cohort study of 26000 children. Acta Psychiatrica Scandinavica 137, 277-286.

Stenbacka M and Jokinen J (2015) Violent and non-violent methods of attempted and completed suicide in Swedish young men: the role of early risk factors. BMC Psychiatry 15, 196.

Stone AA, Schwartz JE, Broderick JE and Deaton A (2010) A snapshot of the age distribution of psychological well-being in the United States. Proceedings of the National Academy of Sciences 107, 9985-9990.

Tang TZ, DeRubeis RJ, Hollon SD, Amsterdam J, Shelton R and Schalet B (2009) Personality change during depression treatment: a placebocontrolled trial. Archives of General Psychiatry 66, 1322-1330.

Wiktorsson S, Berg AI, Billstedt E, Duberstein PR, Marlow T, Skoog I and Waern $\mathbf{M}$ (2013) Neuroticism and extroversion in suicide attempters aged 75 and above and a general population comparison group. Aging \& Mental Health 17, 479-488.

World Health Organization (2012) Public health action for the prevention of suicide: a framework. Geneva, Switzerland: WHO Document Production Services. 